\title{
Evaluation of residual stresses in a tube-to-plate welded joint
}

\author{
Andrea Chiocca $^{1, *}$, Francesco Frendo $^{1}$, and Leonardo Bertini ${ }^{1}$ \\ ${ }^{1}$ University of Pisa, Department of Civil and Industrial Engineering
}

\begin{abstract}
A deep understanding of the manufacturing process is needed in order to achieve safety and quality requirements for parts and components; to this regard, residual stresses play an important role in welded structures. Residual stresses are mainly caused by the extremely severe thermal cycle to which the welded metal and base material are subjected to during welding process and their knowledge leads to a better static and fatigue assessment of welded joints. This work deals with the study of residual stresses for a tube to plate T-joint, made of S355JR carbon steel. The work was carried out by both numerical simulations and experimental tests. The numerical simulations were performed by Ansys FE code through a structural-thermal full transient analysis to evaluate stress, strain and temperature in each node at each step of the simulation. The "birth and death" method was employed, together with temperature-dependent material properties. A 2D and a 3D simulation were performed, in order to evaluate possible differences due to the welding process. Numerical results were compared to some preliminary measurements obtained through an incremental cut made on the plate.
\end{abstract}

\section{Introduction}

Residual stresses are commonly associated with unexpected failures and dimensional inaccuracies. In particular, they affect the component integrity, reducing buckling strength [1], fatigue life [2] and promoting stress corrosion cracking [3]. These stresses commonly occur through different mechanisms: inelastic deformations, temperature gradients, and phase transformation. Sometimes, only one of these mechanisms is significant, while other times they can occur simultaneously; this is the case of welding processes [4].

Residual stresses are detrimental for the integrity and service of the component, for this reason, their evaluation plays an important role. Anyway, through experimental evaluation it is difficult to achieve complete and statistically relevant results [5]. For instance, the detection of residual stresses is based always on indirect methods; to obtain the stress field in a component it is usually necessary to evaluate another quantity, like the elastic strain or the displacement at given points of a specimen or component. Different procedures are commonly used to measure residual stresses: destructive, semi-destructive, non-destructive techniques, e.g. X-ray diffraction, ultrasonic, magnetic or the blind hole drilling method (BHD).

\footnotetext{
*e-mail: andrea.chiocca@phd.unipi.it
} 
The knowledge of the residual stress field in a component may lead to optimized design and, consequently, weight and cost decrease. For instance, the presence of compressive residual stresses is sometimes sought in order to increase the resistance for the surface-related failure (e.g. the laser peening use in turbine engine fan).

Among others, a technique that is becoming commonly used to predict residual stresses is the finite element analysis (FEA) [6-8], being accessible and with a less time effort compared the experimental tests. The finite element method (FEM) is useful in understanding the influence of the different parameters affecting the resulting residual stress field. Nevertheless, it is a challenge to consider all the parameters involved, so approximations in the model have to be introduced.A substantial number of articles dealing with finite element simulations of residual stresses can be found in the technical literature, for both 3D and 2D numerical simulations. In particular, different topics are covered by different authors, such as the influence of welding residual stresses on creep behaviour (Zhou et al. [9]) and the stress prediction for further incorporation in fatigue crack growth analysis (Barsoum et al. [2]), and so on.

The present work is focused on a tube to plate single-pass welding. Residual stresses are commonly generated by the volumetric incompatibilities given by the rapid temperature decrease; consequently, the maximum stress and strain are expected close to the weld seam on the plate surface.

A sequential uncoupled thermal-structural analysis was used, where the structural solution depends only from the thermal one and not vice-versa. The reason for that is the limited displacement during welding that affects the thermal distribution in a negligible way. The sequential uncoupled method is commonly used to study welding process through FE-models as shown in $[4,10]$. The welding process is simulated by the "birth and death" technique combined with an initial nodal temperature of the welding seam, because of its easier controllability and implementation.

\section{Finite element analysis}

The commercial software Ansys was used for the following work. The numerical simulation consists of uncoupled thermal-mechanical full transient analysis. The use of an uncoupled formulation is due to a negligible dimensional variation of the component during welding; this was retrospectively verified.

The simulations were carried on by means of thermal and structural steps, independently solved. Anyway, the analysis was structured in a way to consider the transient temperature field acting on the stress, through the thermal expansion coefficient and the thermalmechanical temperature dependent properties. The entire experimental process was simulated, this consists of the welding process, followed by the specimen's cooling and then the hole generation through an incremental cut, as further described in section 3.

The first step consists of a heat conduction problem followed by a structural analysis where the specimen's thermal history was applied as a load; finally, the holes cutting through the base plate was simulated.

The problem is intrinsically asymmetric due to the welding procedure, and for this reason, a 3D model was assumed necessary to describe the process. The purpose of the 3D model was to get the stress and strain fluctuation along the circumferential direction. However, the 3D model required a very high computational effort because of its non-linearities; for this reason a 2D model was developed as well. A 2D model is easier to manipulate and optimize by varying its parameters, resulting in faster development of the procedure; an example of the two finite element models is shown in figure 1.

Phase transformation effects were not considered, this is due to the lack of information about material properties at high temperature. However, temperature dependent thermal- 
mechanical material properties were included (the reader can refer to section 2.1 and section 2.2 for the complete set of used material properties).

With reference to the specimen geometry, some hypotheses were introduced in the FEmodels:

- the weld seam geometry was simplified by a triangle, even if during the real process part of material flows towards the plate because of the gravity, as shown in figure 1;

- as already stated the "birth and death" was used to approximates the real process; this is usually adopted for FE simulations of the welding process (some examples are reported in $[7,11,12])$;

- cooling of the molten material is simulated by an initial nodal temperature boundary condition instead of a volumetric heat flux. This hypothesis is due to easier controllability of the nodal temperature.
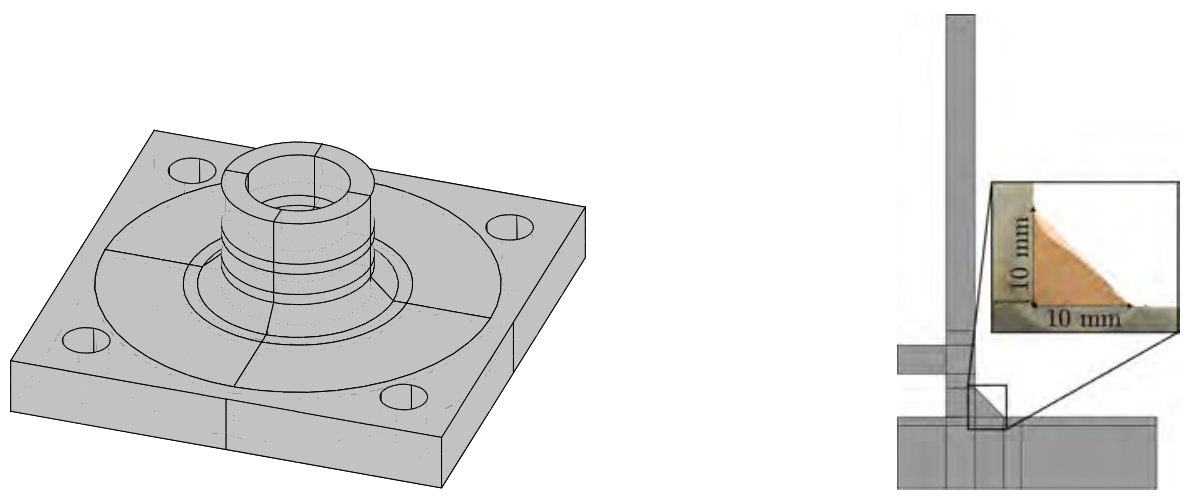

Fig. 1. 3D and 2D FE-models example.

\subsection{Thermal analysis}

The thermal analysis is a transient non-linear simulation. A PLANE55 element was used in the $2 \mathrm{D}$ model, having four nodes with only one degree of freedom, the temperature. The element SOLID70 was employed in the 3D model; it is a brick with eight nodes and one degree of freedom.

The load was defined by applying a fixed initial temperature $\left(T_{i}\right)$ at all the welding seam's nodes. Essentially $T_{i}$ was chosen in two different ways: the melting temperature of the base metal (i.e. the temperature at which the molten metal is expected to be in contact with the plate, $T_{i}=1673 \mathrm{~K}$ ) or the temperature found by infra-red scanning of the welding process done by Farson et al. [13] $\left(T_{i}=3273 \mathrm{~K}\right)$. All the remaining nodes were initialized at the environment temperature $\left(T_{0}\right)$ of $293 \mathrm{~K}$.

The temperature dependent thermal material properties were collected from Bhatti et al. [6]. Both convection and radiation are considered constant during the simulation; the convection was set to a value of $21 \mathrm{~W} \mathrm{~m}^{-2} \mathrm{~K}^{-1}$, while the radiation was set at 0.79 at ambient temperature. The convection is significant for lower temperatures than radiation, instead, for temperatures close to the material melting point, radiation is significant. 


\subsection{Structural analysis}

Similarly, even the mechanical structural analysis is a transient non-linear simulation. The same mesh and geometry of the thermal simulation was used, anyway, the elements were updated. The PLANE182 element was adopted for the 2D model, while the SOLID185 was used for the 3D one. In this case, the degrees of freedom for the analysis are the displacement at the nodes instead of temperatures, i.e. two degrees of freedom (x-y) for the four nodes PLANE182 and three degrees of freedom (x-y-z) for the 8 nodes SOLID185.

The welding was executed by rotating the tube and plate around the tube axes, leaving the component free to move. Because of this, in the FE-model only the upper tube surface was fixed in the radial and hoop directions. Although the boundary conditions (BC's) were not related to the real process constraints, these were aimed to leave the specimen free to move in the weld region. Basically, the edge effect given by the constraints was dissipated in less than a tube thickness and, as regards the effect of the BC's on the stress-strain field, this is negligible.

Likewise, the thermal simulation, the element "birth and death" technique was applied with the same sequence to simulate the welding process. The load consisted of a thermal body force applied at each node for each time-step. The temperatures were obtained from a binary file resulting from the thermal simulation. Through the thermal expansion coefficient, the software calculated the deformation of each element. The thermal strain is given by

$$
\varepsilon^{t h}=\alpha(T)\left(T-T_{r e f}\right)
$$

where $\varepsilon^{\text {th }}$ is the structural thermal strain, $\alpha(T)$ the instantaneous coefficient of thermal expansion, $T$ the element evaluation temperature and $T_{r e f}$ is the temperature at which zero thermal strain exists.

Hence, it was necessary to define two different materials' reference zero strain temperatures: a material for the tube and plate with a reference temperature of $293 \mathrm{~K}$, one material for the welding seam with a reference temperature of $1673.15 \mathrm{~K}$ or $3273 \mathrm{~K}$ respectively.

The thermal expansion coefficient was taken from Bhatti et al. [6], while a multilinear elastic-plastic material law developed by Chen et al. [14] was implemented. The Poisson's ratio was kept constant at a value of 0.3 . In general, it is common not to consider its variation like already done by $[2,6,8,10]$.

\section{Experimental procedure}

A tube to plate welded joint of S355JR was studied, as shown in figure 2. The specimen was composed by a tube of $44 \mathrm{~mm}$ of internal diameter and a thickness of $10 \mathrm{~mm}$, this was welded at a square plate of $190 \mathrm{~mm}$ side and $25 \mathrm{~mm}$ of thickness. Four holes with a diameter of $21 \mathrm{~mm}$ were executed symmetrical on the plate as well as one $10 \mathrm{~mm}$ hole in the center of the plate. The holes were necessary for the experimental fatigue tests set up not describe in this work. Furthermore, a reinforcement was placed inside the tube at a distance of $15 \mathrm{~mm}$ from the plate surface.

Both the reinforcement welding and the tube-to-plate welding were manufactured by a singlepass of gas metal arc welding (or GMAW). Anyhow only the tube to plate welding was considered in the following. This was obtained by rotating the tube and plate around the tube axes and keeping fixed the welding torch.

In table 1 the experimentally tested specimens are summarized. It is given a description of post-production processes, number of strain gauges and their position on the plate surface. 


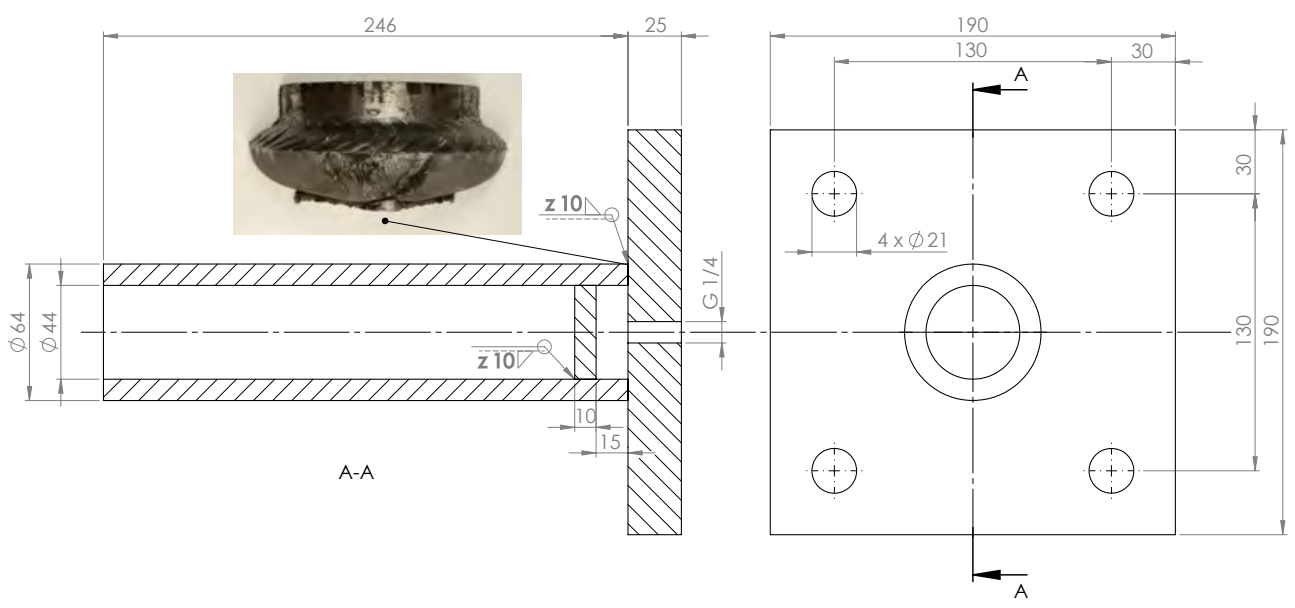

Fig. 2. Geometric dimension of the specimen and fractured surface after fatigue test.

Table 1. Experimental test summary

\begin{tabular}{ccccc}
\hline Name & Post-production & Strain gauges & Radius $(\mathrm{mm})$ & Angle $\left(^{\circ}\right)$ \\
\hline Specimen-1 & None & 8 & $6.5 / 9 / 13$ & 360 with step of 90 \\
\hline Specimen-2 & None & 8 & $6.5 / 9 / 13$ & 360 with step of 90 \\
\hline Specimen-3 & None & 12 & 6.5 & 360 with step of 30 \\
\hline Specimen-4 & Annealing & 12 & 6.5 & 360 with step of 30 \\
\hline
\end{tabular}

In order to validate FEM results, residual strains were evaluated on the plate surface through incremental holes cutting on the back of the plate, as shown in figure 3. The holes were created on the bottom surface of the plate with a step-increment of $5 \mathrm{~mm}$; this process releases residual stresses because of the elimination of material from the specimen. Several strain gauges were placed around the weld seam to detect radial strain, as shown in figure 3 . During the hole cutting the strain gauges were able to detect the plate deformations given by redistribution of stresses.

The procedure is composed by two holes of different diameters and depths, the first one is a through-hole of $42 \mathrm{~mm}$ diameter, while the second one has a maximum depth of $20 \mathrm{~mm}$ and a diameter of $100 \mathrm{~mm}$.

\section{Results and discussion}

Different simulations were carried out during this study as described below.

Strain measurements in radial direction were done through strain gauges, according to table 1. Furthermore, results from 2D and 3D numerical analysis were evaluated.

As it can be seen from figure 4 and figure 5 both temperature and material properties have been found to be parameters of influence for the numerical analysis. Figure 4 shows the strain resulting from the difference between the strain obtained after holes cutting and the strain obtained after cooling, this for different $T_{i}$ of the welding seam. It can be noticed that, as the starting temperature increases, the maximum residual strain decreases. A higher $T_{i}$ 


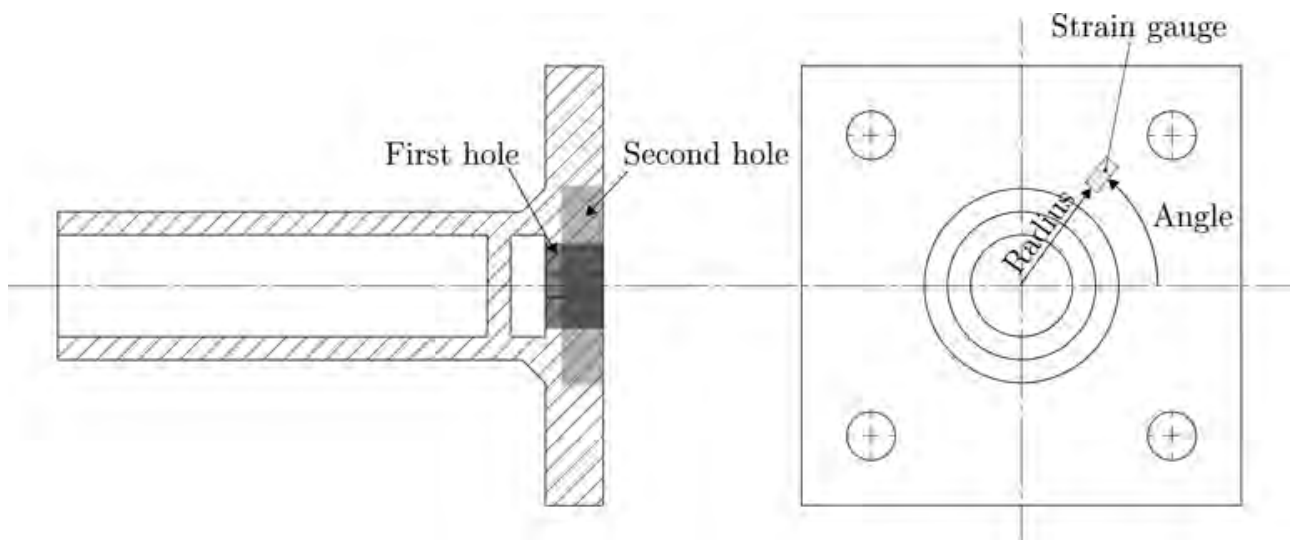

Fig. 3. Hole creation method to detect residual stresses on the plate surface and strain gauges positioning over the plate surface.

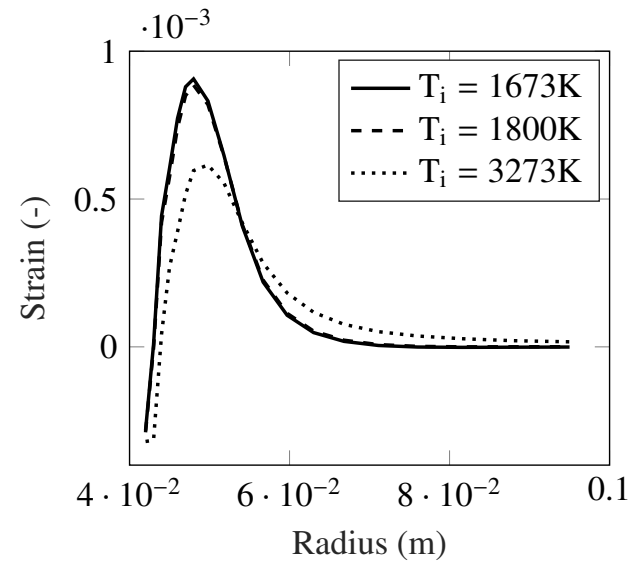

Fig. 4. 2D simulation results comparison for different initial temperatures $\left(T_{i}\right)$ of the welding seam.

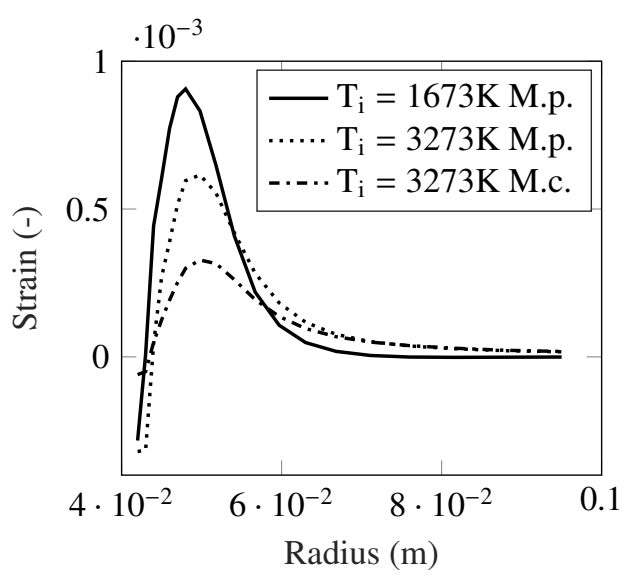

Fig. 5. 2D simulation results comparison for different initial temperatures $T_{i}$ and material properties (M.p. = Partial multi linear elasticplastic, M.c. = Complete multi linear elasticplastic)

heats up more material around the welding seam, however, the volumetric changes resistance of the material is weakened by the poor material properties at high temperature, leading to lower stresses and strains. For instance, figure 5 can be explained in the same way; where "M.p." refer to a partial set of multilinear elastic-plastic temperature dependent material properties (i.e. they are described until a temperature of $933 \mathrm{~K}$ ), while "M.c." refers to a complete set of multilinear elastic-plastic temperature dependent material properties (i.e. the complete set used by Chen et al. [14]). In this case, the use of a complete set of elastic-plastic material properties further weakens the resistance of the material for volumetric changes. Basically, when the material is at temperatures higher than $933 \mathrm{~K}$, the numerical program implements much lower yield strength and elastic modules that lead to lower residual strains. 

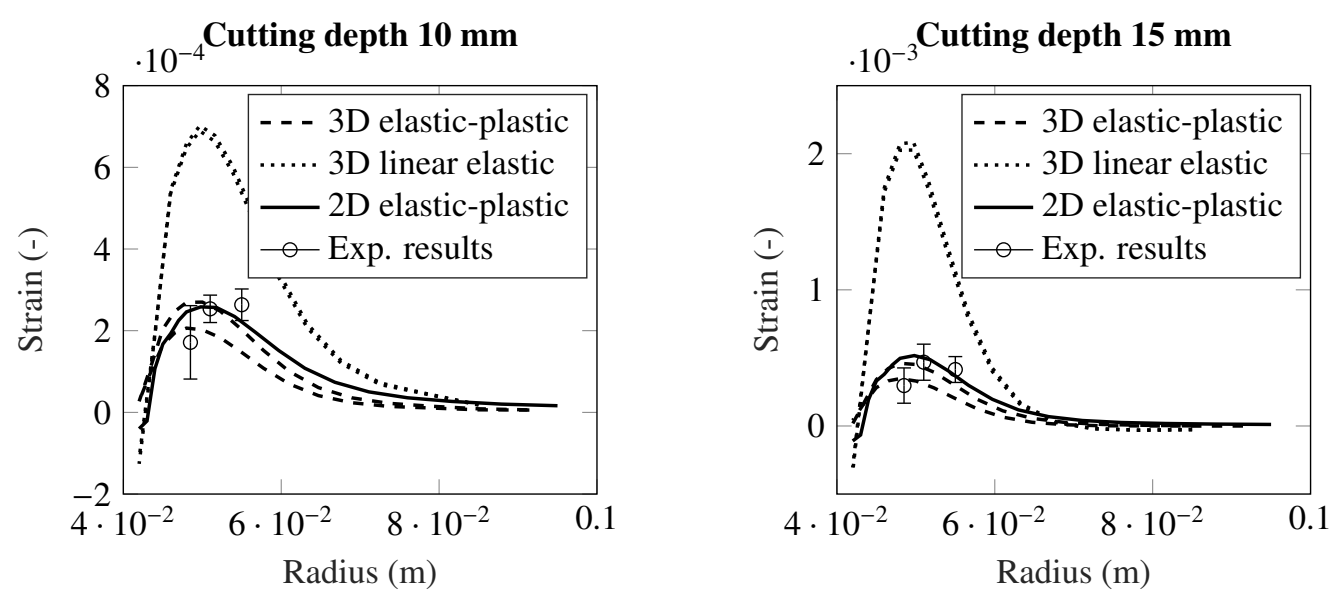

Fig. 6. Comparison between numerical and experimental results for different cutting depth.

In figure 6 all the main results are shown. All the results are obtained for an initial welding seam's temperature of $T_{i}=1673 \mathrm{~K}$. In particular, the 3D analyses reported the entire range of strain solutions caused by the stress and strain variance along the circumference. It can be noticed that the variation between the maximum and minimum strain is not remarkable for both the linear-elastic and elastic-plastic 3D simulations. Actually, for the 3D linear elastic analysis, the maximum relative percentage difference between the maximum and minimum strain is $2.7 \%$, while for the elastic-plastic model is $26 \%$.

The experimental results are described through the mean value and standard deviation of all the specimens for different radius.

The 3D linear elastic simulation with temperature dependent material properties overestimates the experimental results as expected. The 3D and 2D elastic-plastic models with temperature dependent material properties lead to a better result compared the previous one.

On average, the 2D simulation is easier to be setup. The 3D analysis may be optimized, considering the additional parameters with respect to the $2 \mathrm{D}$ simulation, such as the total welding time, the "birth and death" method, the starting weld seam temperature. Consequently, a further study would be needed in order to research the optimum set of parameters in a $3 \mathrm{D}$ elastic-plastic simulation.

Ultimately, the von Mises stress field, resulting from the 2D elastic-plastic simulation, is shown in figure 7. As expected, there is a strong stress gradient in the weld seam area, with a maximum value on the apex of the weld seam. In particular, the maximum value of stress is close to the yield strength of the base material, this result does not differ from the common practice and from many other studies on residual stress. In figure 8 a comparison between the radial and hoop stress of the $3 \mathrm{D}$ and $2 \mathrm{D}$ simulations is given. All the results are obtained for an initial welding seam's temperature of $T_{i}=1673 \mathrm{~K}$. As always, regarding the $3 \mathrm{D}$ analysis, the circumferential stress range is plotted; the difference between the two analyses is in the same order of magnitude of the difference for the strain results.

\section{Conclusions}

In this study, both numerical and experimental results are employed to investigate the residual strain field near the welding seam in a tube-to-plate welded joint. The comparison between 


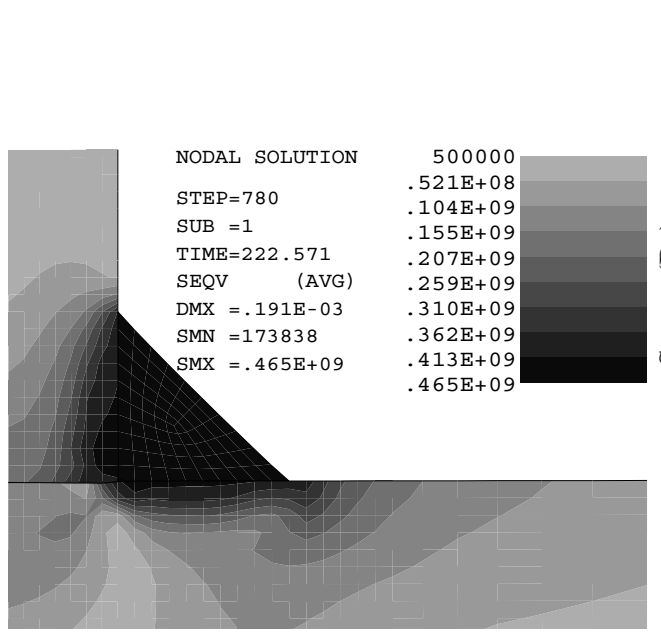

Fig. 7. Von Mises stress map in the $2 \mathrm{D}$ simulation.

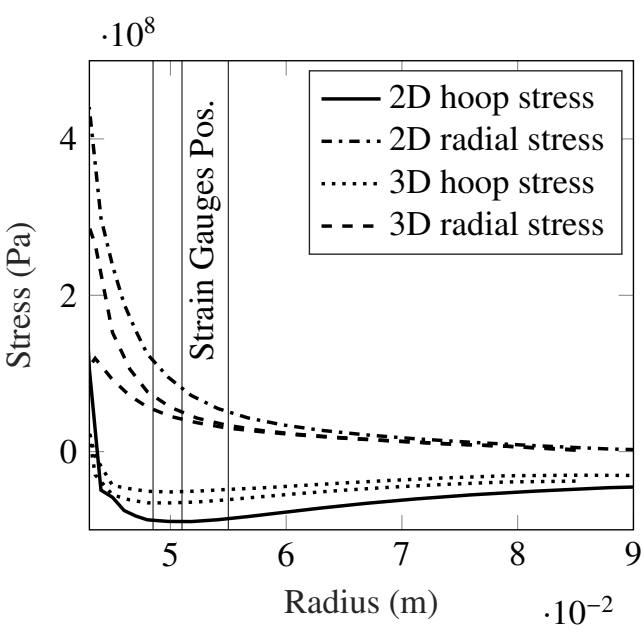

Fig. 8. Radial and hoop stress comparison for $2 \mathrm{D}$ and $3 \mathrm{D}$ analysis. The stress interval is shown for the 3D simulation results.

the results is applied in order to predict and verify residual strains and stresses found by FEM. The following conclusions can be drawn:

- a proper estimate of parameters such as welding seam's initial temperature and elasticplastic material properties is necessary in order to have a good estimation of the strain and stress field;

- the $2 \mathrm{D}$ axisymmetric elastic-plastic model can predict in a fairly satisfactory way the strain field;

- the variation of stress and strain in the circumferential direction near the weld seam can be neglected in a first order analysis, both for the linear-elastic and elastic-plastic analysis; basically, the asymmetry introduced by the welding process is minor;

- the difference between the two models is so small that, considering the overall effort necessary for the $3 \mathrm{D}$ analysis, the use of the $2 \mathrm{D}$ model is preferable. 


\section{References}

[1] M. Clarin, High strength steel : local buckling and residual stresses (2004)

[2] Z. Barsoum, A. Lundbäck, Simplified FE welding simulation of fillet welds - 3D effects on the formation residual stresses, Engineering Failure Analysis 16, 2281 (2009)

[3] G. Vanboven, W. Chen, R. Rogge, The role of residual stress in neutral pH stress corrosion cracking of pipeline steels. Part I: Pitting and cracking occurrence, Acta Materialia 55, 29 (2007)

[4] D. Deng, FEM prediction of welding residual stress and distortion in carbon steel considering phase transformation effects, Materials \& Design 30, 359 (2009)

[5] G.A. Alpsten, Tech. rep., Thermal residual stresses in hot-rolled steel members, Fritz Laboratory Reports (1968)

[6] A.A. Bhatti, Z. Barsoum, H. Murakawa, I. Barsoum, Influence of thermo-mechanical material properties of different steel grades on welding residual stresses and angular distortion, Materials \& Design (1980-2015) 65, 878 (2015)

[7] A. Capriccioli, P. Frosi, Multipurpose ANSYS FE procedure for welding processes simulation, Fusion Engineering and Design 84, 546 (2009)

[8] J. Cho, B. Lee, Y. Moon, C.V. Tyne, Investigation of residual stress and post weld heat treatment of multi-pass welds by finite element method and experiments, Journal of Materials Processing Technology 155-156, 1690 (2004)

[9] Y. Zhou, X. Chen, Z. Fan, S. Rao, Finite Element Modelling of Welding Residual Stress and Its Influence on Creep Behavior of a 2.25Cr-1Mo-0.25V Steel Cylinder, Procedia Engineering 130, 552 (2015)

[10] D. Deng, H. Murakawa, Prediction of welding residual stress in multi-pass butt-welded modified 9Cr-1Mo steel pipe considering phase transformation effects, Computational Materials Science 37, 209 (2006)

[11] T.L. Teng, P.H. Chang, W.C. Tseng, Effect of welding sequences on residual stresses, Computers \& Structures 81, 273 (2003)

[12] D. Deng, H. Murakawa, W. Liang, Numerical and experimental investigations on welding residual stress in multi-pass butt-welded austenitic stainless steel pipe, Computational Materials Science 42, 234 (2008)

[13] D. Farson, R. Richardson, X. Li, Infrared measurement of base metal temperature in gas tungsten arc welding, Welding Journal 77 (1998)

[14] J. Chen, B. Young, B. Uy, Behavior of High Strength Structural Steel at Elevated Temperatures, Journal of Structural Engineering 132, 1948 (2006) 